HORTSCIENCE 26(8):1069-1072. 1991.

\title{
Regeneration of Brassica oleracea from Peduncle Explants
}

\author{
Mary C. Christey ${ }^{1}$ and Elizabeth D. Earle \\ Plant Breeding Department, Cornell University, Ithaca, NY 14853-1902
}

Additional index words. broccoli, cabbage, cauliflower, Chinese broccoli, rapid-cycling Brassica oleracea, in vitro propagation

\begin{abstract}
Peduncle explants from 12 Brassica oleracea L. lines representing five varieties [broccoli (italica), cabbage (capitata), cauliflower (botrytis), Chinese broccoli (alboglabra), and rapid-cycling B. oleracea] readily regenerated shoots in vitro. Average regeneration rates of more than $75 \%$ were obtained for most lines, with up to 35 shoots per explant. Shoots were visible within 7 to 10 days. Initial regeneration was polarized, occurring mainly from the basal end of explants. Linsmaier-Skoog-based medium containing $1 \mathrm{mg} \mathrm{BA}$ /liter was suitable for shoot regeneration from all 12 lines tested. Plants were rooted on hormone-free medium and transferred to soil. Chemical name used: benzyladenine (BA).
\end{abstract}

Brassica oleracea is an economically important vegetable species, containing 14 vegetable varieties, including broccoli, cabbage, 'cauliflower, and Chinese broccoli. Practically every part of the plant can be used, including the leaves (cabbage), axillary buds (brussels sprouts), stems (kohlrabi), floral primordia (cauliflower), and flower buds (broccoli). These vegetables are amenable to in vitro manipulation; shoot regeneration has been demonstrated for almost all varieties using explants from various vegetative organs, and also from floral organs (reviewed in Christey, 1989, and in Zee and Johnson, 1984).

The use of peduncle explants for the regeneration of Brassica was first reported in B. napus by Stringam (1977). Regeneration

Received for publication 12 July 1990. Support for this work was provided by a National Research Advisory Council fellowship from the New Zealand government to M.C.C. and from U.S. Dept. of Agriculture grant 85-CRCR-1-1608 to E.D.E. The cost of publishing this paper was defrayed in part by the payment of page charges. Under postal regulations, this paper therefore must be hereby marked advertisement solely to indicate this fact. 'Current address: Dept. of Scientific and Industrial Research, Crop Research Division, Private Bag, Christchurch, New Zealand. frequencies of more than $80 \%$ were obtained with multiple buds per explant. Peduncle explants have since been used for regeneration and transformation of B. napus (Fry et al., 1987), but not for B. oleracea. Although previous studies have demonstrated shoot regeneration from many varieties of $B$. oleracea, rates of shoot regeneration and number of shoots per explant were generally low. We aimed to determine whether peduncle explants could be used to obtain high levels of shoot regeneration with $B$. oleracea and, if so, whether the procedure was suitable for several $B$. oleracea varieties. Shoot regeneration from peduncle explants of $12 \mathrm{~B}$. oleracea lines representing five botanical varieties is reported here. The use of this regeneration system for transformation and in vitro propagation of desirable genotypes is discussed.

Plant material. The plant materials used in this study are described in Table 1 . The four vegetables (Chinese broccoli, cauliflower, cabbage, and broccoli) were chosen to represent a range of $B$. oleracea varieties grown in the northeastern United States. Lines were also included to permit comparison of cytoplasm effects and to test regeneration from rapid-cycling (RC) B. oleracea (Williams, 1985).

Peduncle explants from broccoli and Chinese 
Table 1. Brassica oleracea lines used as sources of peduncle explants.

\begin{tabular}{llllll}
\hline Variety & Common name & Cultivar or line & Cytoplasm & \multicolumn{1}{c}{ Description } & Reference or source \\
\hline Alboglabra & Chinese broccoli & Guy Lon & F & & Fredonia Seeds, Fredonia, N.Y. \\
Botrytis & Cauliflower & NY 7642 B & F & Inbred line & Dickson, 1985 \\
& Cauliflower & $883824 / 883825$ & ATR, F & Cybrids from NY 7642A & Jourdan et al., 1989 \\
Capitata & Cabbage & Unknown & F & & Plantations, Cornell Univ., Ithaca, N.Y. \\
Italica & Broccoli & Packman & F & Hybrid & Fredonia Seeds \\
& Broccoli & Green Comet (GC) & F & F, hybrid & Harris Moran Seed Co., Rochester, N.Y. \\
& Broccoli & GCR & F & GC regenerant & Robertson and Earle, 1986 \\
& Broccoli & $84-3185$ & CMS, ogura & Derived from GC & y \\
& Broccoli & BN\#4 & CMS, nigra & Derived from GC & y,x \\
Rapid cycling & Broccoli & BN\#5 & CMS, nigra & Derived from GC & y,x \\
\hline
\end{tabular}

${ }^{{ }^{2} \mathrm{ATR}}=$ atrazine-resistant chloroplasts; $\mathrm{F}=$ male fertile; $\mathrm{CMS}=$ cytoplasmic male sterile.

yDeveloped by M. Dickson, New York State Agricultural Experiment Station, Geneva.

×BN\#4 and BN\#5 materials included two original plants as well as plants regenerated from leaf protoplasts from them. The two sources of plants were not distinguished for this work.
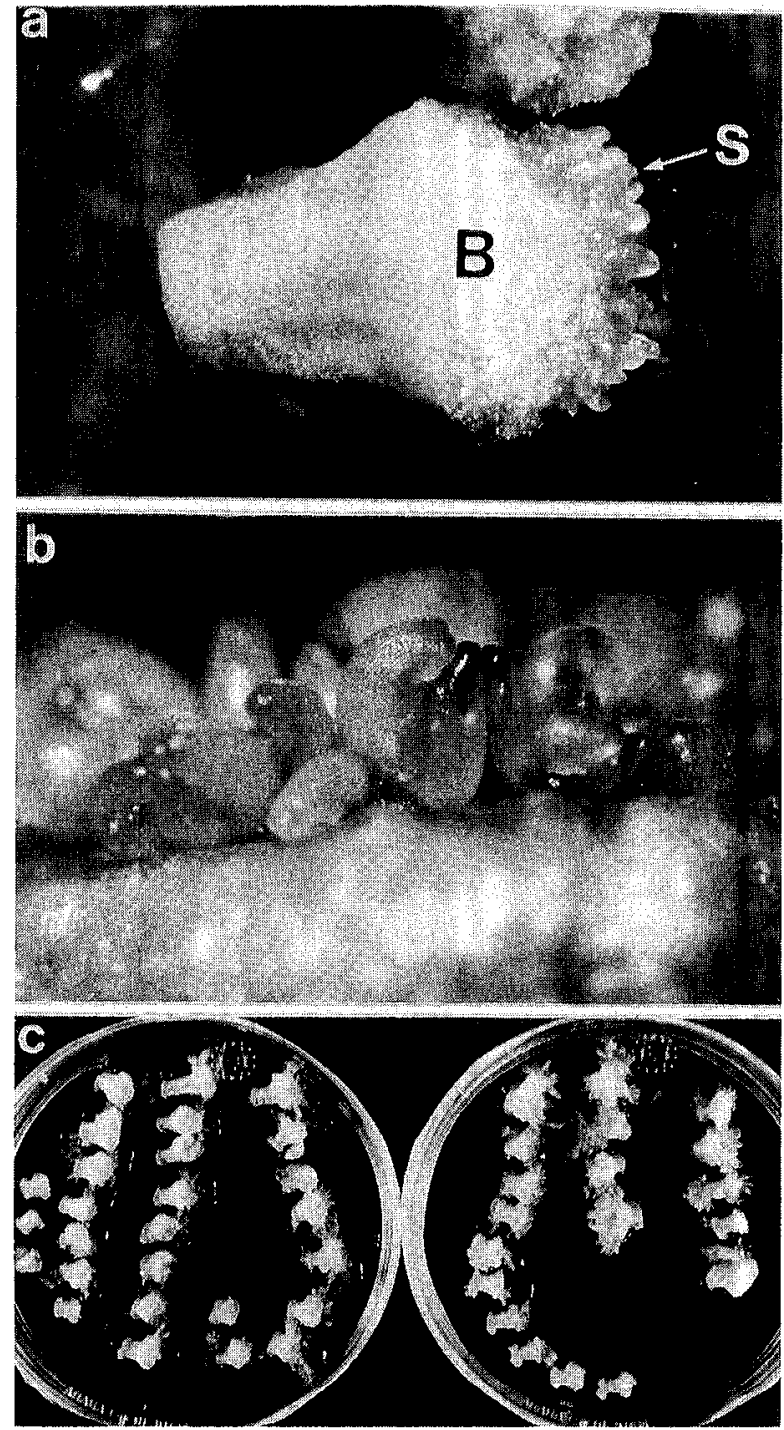

Fig. 1. Shoot regeneration from peduncle explants. (a) Broccoli peduncle explant after 8 days in culture. Note the swollen basal end (B) with shoot buds (S) ( $\times 5$ ). (b) Shoot buds on a Chinese broccoli peduncle explant after 10 days in culture $(\times 20)$. (c) Cauliflower peduncle explants after 14 days in culture $(x 0.5)$.

broccoli were obtained from plants grown in a greenhouse at 25 to $30 \mathrm{C}$ under 24 -h illumination (daylight supplemented with coolwhite fluorescent lights, $\left.100 \mu \mathrm{mol} \cdot \mathrm{m}^{-2} \cdot \mathrm{s}^{-1}\right)$. Cabbage plants were grown in the field, and cauliflower plants were initially grown in the field and then transplanted to the greenhouse at maturity. Explants from RC B. oleracea were obtained from plants grown in a greenhouse at 18 to $25 \mathrm{C}$ under 12 -h illumination (daylight supplemented with mercury vapor lamps, $\left.100 \mu \mathrm{mol} \cdot \mathrm{m}^{-2} \cdot \mathrm{s}^{-1}\right)$.
Culture of peduncle explants. Peduncles were collected from plants that were bolting. The peduncles chosen had closed flower buds and few, if any, open buds. Only the top 5 to $7 \mathrm{~cm}$ was used to avoid the harder, woody lower tissue. Flower buds and pedicels were discarded.

Peduncles were surface sterilized by immersion in $70 \%$ ethanol for 3 to $5 \mathrm{~min}$ and then $0.5 \%$ sodium hypochlorite for 10 to 15 min, followed by three 5-min rinses with sterile water. Tissue damaged by the bleach was removed before cutting each peduncle into five to ten 5-mm explants. Explants were placed horizontally onto Linsmaier-Skoog (LS) medium (Linsmaier and Skoog, 1965) containing 3\% sucrose and $1 \mathrm{mg}$ BA/liter and solidified with $0.25 \%$ Gelrite (Scott Laboratories, Carson, Calif.). The order and orientation (basal vs. apical) of each explant was noted. About 20 to 25 explants were cultured per $9-\mathrm{cm}$ petri plate,

After 3 to 4 weeks, the entire regenerating ends of some explants were excised and transferred to hormone-free LS medium (LS-N). Individual shoots $(5-10 \mathrm{~mm})$ were excised 10 to 14 days later and placed on LS-N for rooting. After transfer to soil, high humidity was maintained either by placing plants in a mist unit or by covering them with a plastic bag. After 1 to 2 weeks, the plants were either removed from the mist unit or holes were cut in the bag before its removal 1 week later.

RC B. oleracea cultures were maintained at $25 \mathrm{C}$ under a $16-\mathrm{h}$ photoperiod provided by cool-white fluorescent lights, 80 $\mu \mathrm{mol} \cdot \mathrm{m}^{-2} \cdot \mathrm{s}^{-1}$. All other cultures were maintained at $25 \mathrm{C}$ under a 16 -h photoperiod provided by an equal number of cool-white and Gro and Sho (General Electric, Schenectady, N.Y.) fluorescent lights, 45-70 $\mu \mathrm{mol} \cdot \mathrm{m}^{-2} \cdot \mathrm{s}^{-1}$. Plants in soil were grown at 25C under a 16-h photoperiod with lighting from cool-white fluorescent lights, 35 $\mu \mathrm{mo} 1 \cdot \mathrm{m}^{-2} \cdot \mathrm{s}^{-1}$. After 3 to 4 weeks, some plants were transferred to the greenhouse as described above.

Shoot regeneration was readily obtained from peduncle explants of all lines tested (Table 2). Average regeneration frequencies were high, ranging from $41 \%$ to $98 \%$ but usually $>75 \%$. Buds per explant were also high, ranging from one to 35 and generally 

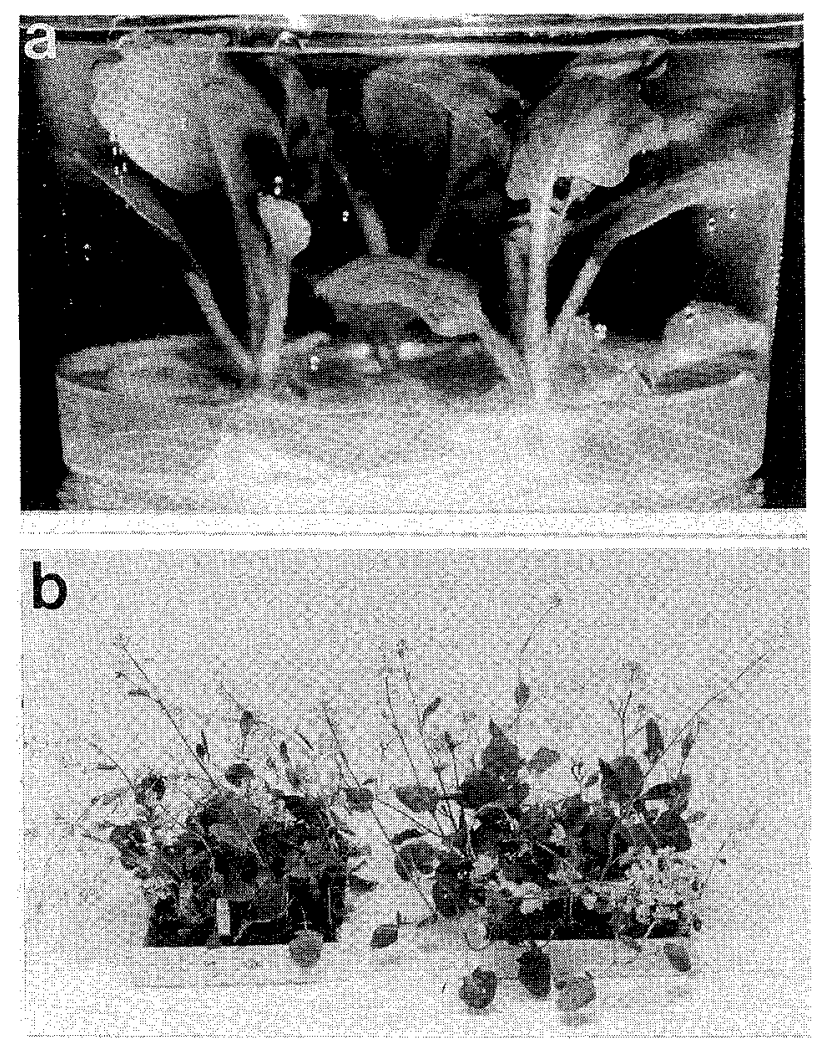

Fig. 2. Plant development. (a) Rapid cycling (RC) Brassica oleracea plants 3 weeks after transfer to hormone-free medium $(\times 0.9)$. (b) RC B. oleracea plants 3 weeks after transfer to soil $(\times 0.06)$.

Table 2. Shoot regeneration from peduncle explants of various Brassica oleracea materials. ${ }^{2}$

\begin{tabular}{|c|c|c|c|c|}
\hline $\begin{array}{l}\text { Variety, } \\
\text { cultivar, or line }\end{array}$ & $\begin{array}{l}\text { Explants } \\
\text { cultured }\end{array}$ & No. replicates ${ }^{y}$ & $\begin{array}{l}\text { Percentage } \\
\text { regeneration } \\
( \pm \mathrm{SD})\end{array}$ & $\begin{array}{c}\text { Buds/explant } \\
\text { (range) }\end{array}$ \\
\hline \multicolumn{5}{|l|}{ Alboglabra } \\
\hline Guy Lon & 196 & 22 & $95 \quad(7.4)$ & $13(2-21)$ \\
\hline \multicolumn{5}{|l|}{ Botrytis } \\
\hline 883824 & 154 & 23 & $86(18.1)$ & $7(1-20)^{x}$ \\
\hline 883825 & 132 & 16 & $87(14.8)$ & $12(1-35)$ \\
\hline NY 7642B & 86 & 12 & $78(14.7)$ & $6(1-30)$ \\
\hline \multicolumn{5}{|l|}{ Capitata } \\
\hline Cultivar unknown & 97 & 8 & $85(16.3)$ & $11(1-25)^{x}$ \\
\hline \multicolumn{5}{|l|}{ Italica } \\
\hline Packman & 152 & 22 & $61(25.2)$ & $6(1-20)$ \\
\hline Green Comet & 56 & 8 & $98(7.1)$ & $9(1-26)$ \\
\hline GCR & 243 & 21 & 94 (11.5) & $7(3-14)$ \\
\hline $84-3185$ & 438 & 31 & $41(31.8)$ & $\mathrm{ND}^{\mathrm{w}}$ \\
\hline BN\#4 & 694 & 49 & $78(27.2)$ & ND \\
\hline BN\#5 & 655 & 48 & $79(23.8)$ & ND \\
\hline \multicolumn{5}{|l|}{ Rapid-cycling } \\
\hline CrGC\#3 & 164 & 17 & $88(16.1)$ & $13(1-30)^{x}$ \\
\hline
\end{tabular}

${ }^{2}$ Determined after 28 days, except where noted.

'Each replicate consists of the explants from one peduncle.

'Determined after 14 days.

"Not determined.

more than 10 per explant. Shoots were usually visible after 7 to 10 days. The same medium could be used for all lines tested.

Effect of culture conditions. In preliminary experiments, $0.8 \%$ Bacto Agar (Difco, Detroit, Mich.) was used to solidify the medium, but browning of the explants in the region in contact with the medium was noted. Such browning did not occur with Gelrite, which was routinely used in further work.

Initially, explants were cultured vertically with the basal end in contact with the medium, as done by Stringam (1977) and Fry ered by a small amount of white or palegreen callus. The surface area of the basal end was about twice that of the apical end. After 28 days, the surface area of the basal end (6-8 $\mathrm{mm}$ in diameter) was about five times that of the apical end (2-4 mm). Fresh weight of the explants increased dramatically during the experiment, from 40 to $50 \mathrm{mg}$ to 400 to $600 \mathrm{mg}$ after 28 days,

The first signs of regeneration were slightly raised areas on the lower edge of the end of the explants. These areas preceded the appearance of buds which then pushed through from under the surface callus. Shoot regeneration was first noted 7 to 10 days after culture of explants (Fig. $1 \mathrm{a}$ and b). After 2 weeks, numerous vegetative buds were present. Shoot formation was initially polarized: buds were concentrated on the basal end of explants in the region closest to the medium (Fig. la), except on 'Guy Len' explants, which showed initial regeneration from the apical end. Often more than $80 \%$ of the explants regenerated shoots (Table 2, Fig. 1c). Root formation was only rarely noted. By the end of the experiment (day 28), there was no further increase in the number of regenerating explants, but the number of buds per explant had increased and shoots had elongated. Shoots often developed from both ends, although bud number was still usually higher on the basal end. While polarity in the shoot regeneration pattern per explant was noted, there was little effect of original explant position within a peduncle.

Shoot buds were usually pale green with slight reddening of the tips for some lines. Buds from the cauliflower lines were deep red for the first 2 to 3 weeks of culture but turned green as the shoot tips elongated.

Transfer to soil. After 4 weeks, when shoots had elongated, at least 10 shoots from most lines were excised and transferred to LS-N medium. Within 7 days, several short roots had developed from the cut end of more than half the shoots. After 3 to 4 weeks, more than $80 \%$ of the cuttings showed sufficient root and shoot growth for transfer out of culture (Fig. 2a). More than $95 \%$ of these were successfully transferred to soil, but most were not kept for further growth. Plants from $\mathrm{BN \# 4}$ and $\mathrm{BN \# 5}$ peduncle explants were grown to maturity and showed no obvious morphological changes.

From the RC line, 166 plants were transferred to soil, with $98 \%$ surviving to maturity (Fig. 2b). These plants retained the RC phenotype, with flower buds visible 14 days after transfer to soil. Plants were fertile and set seed. All appeared morphologically normal, except for two plants $(1.2 \%)$ that had cream-colored flowers instead of yellow ones. Sectoring of color within individual petals and flowers was apparent on these two plants.

Effect of cultivar on regeneration. The effect of cultivar was studied for broccoli where shoot regeneration percentages for 'Packman' were lower than those for 'Green Comet' (Table 2). In one experiment with 'Packman' peduncle explants, raising the BA level from 1 to 2 or $5 \mathrm{mg} \cdot$ liter $^{-1}$ increased the percentage of explants that formed shoots from $68 \%$ 
to $83 \%$ or $94 \%$, respectively. Although the number of explants per treatment was small (16-23), it appears that an increase in BA can increase regeneration to levels comparable to those in other lines. In $B$. napus, raising the $\mathrm{BA}$ level to $10 \mathrm{mg} \cdot \operatorname{liter}^{-1}$ also increased the percentage of peduncle explants regenerating shoots (data not shown).

Effect of cytoplasm on regeneration. The effect of cytoplasm on regeneration was tested by comparing regeneration from two fertile and three cytoplasmic male-sterile (CMS) broccoli lines, all with 'Green Comet' nuclear backgrounds. These lines contained either the $B$. oleracea cytoplasm ('Green Comet' and 'Green Comet' regenerant), the ogura cytoplasm from Raphanus sativus (84-3185), or the B. nigra cytoplasm (BN\#4 and $\mathrm{BN \# 5);} \mathrm{the} \mathrm{latter} \mathrm{two}$ cytoplasms confer male sterility. The fertile lines had higher percentages of regeneration than either type of CMS line (Table 2). Material with the $B$. nigra cytoplasm showed more shoot regeneration than the ogura line, and experiments were more reproducible. Raising the BA level did not increase the percentage of CMS explants with shoot regeneration.

Our results indicate that peduncle explants from the five tested $B$. oleracea varieties readily regenerate shoots. Regeneration was rapid with multiple shoots per explant. Average regeneration percentages were high; only two lines, 'Packman' and 84-3185, had average regeneration percentages below $75 \%$. The medium used for regeneration from peduncle explants of B. napus (Fry et al., 1987; Stringam, 1977) could also be used for all $B$. oleracea lines tested. Optimum regeneration from leaf discs of $B$. campestris, $B$. oleracea, and $B$. napus required a different hormone combination for each species (Dunwell, 1981).

The regeneration percentages and shoot production per explant and growth patterns obtained here for $B$. oleracea lines are similar to those reported for B. napus by Stringam (1977) and Fry et al. (1987). In contrast, only rare shoot regeneration was obtained from peduncle explants from three RC $B$. campestris lines cultured on this medium and more than 50 other combinations of growth regulators (mainly BA and NAA) (Christey, 1989). These observations of differential regeneration from peduncle explants of $B$. oleracea, B. campestris, and their amphidiploid derivative $B$. napus confirm previous studies in which regeneration of $B$. oleracea and $B$. napus was also higher than that of $B$. campestris (Dietert et al., 1982; Dunwell, 1981; Narasimhulu et al., 1988).

The culture of intact peduncle explants in this study with $B$. oleracea and previously with B. napus (Fry et al., 1987; Stringam, 1977; M.C.C., unpublished observations) produced only vegetative shoots. The plants regenerated required the normal growth period to induce flowering even though the explants were obtained from the flowering zone.

In these experiments, regeneration was first seen on one end of the explants, usually the basal end. Such polarity was also noted with B. napus var. oleifera cv. Oturu peduncle explants cultured in a similar manner (data not shown). There was no corresponding polarity of root regeneration from the opposite end. In contrast, Lazzeri and Dunwell (1986) observed strong polarity of shoot and root regeneration from root and hypocotyl explants of 'Green Comet' broccoli.

A disadvantage of using peduncle explants for shoot regeneration is the time required to obtain them. The use of the RC B. oleracea line, from which peduncle explants were available 6 weeks after planting seed, overcame this disadvantage, allowing evaluation of regenerants for morphology and fertility 10 to 12 weeks after culture of explants. Peduncle explants from RC B. oleracea could thus be used as a model system for the production and study of transgenic plants.

There are several advantages to using peduncle explants for in vitro culture and regeneration. The culture procedure is simple, as one medium was suitable for all lines tested. Numerous explants can be obtained from a plant nondestructively, allowing continued growth of the plant for collection of additional explants or for further evaluation and seed set. In B. oleracea, the only other explants showing comparable rates of regeneration are derived from pedicels (Anderson and Carstens, 1977). The use of both pedicel and peduncle explants could enable a further increase in the shoots recovered from a single peduncle.

Peduncle explants could be useful for the in vitro propagation and rapid multiplication of important or novel genotypes. To be successful such a system has the following requirements: 1) multiple buds per explant and high regeneration, 2) short time from culture to potted plant, 3) reproducibility, and 4) high survival after transplanting from culture to soil.

These features have been met in the peduncle regeneration system reported here for B. oleracea. From a single plant, it is possible to obtain $>150$ explants; assuming a regeneration percentage of $75 \%$ with 10 buds per explant, >1125 shoots could be regenerated from one plant. A further increase in the propagation rate per plant could be obtained by including pedicel explants. The regeneration time is short-only 10 to 12 weeks from initial explant to potted plants. Survival on transfer to soil is high (>95\%) provided care is taken to ensure a gradual decrease in humidity. In addition, little callus is formed before shoot formation, so it is likely that cytological aberrations and somaclonal variation will be slight.

Peduncle explants could also be used for transformation of $B$. oleracea via cocultivation of explants with Agrobacterium tumefuciens or by use of particle acceleration methods. The high percentages of regeneration, with quick production of numerous shoots, mean that transgenic plants might be rapidly obtained, as has been done in $B$. $n a$ pus (Fry et al., 1987).

\section{Literature Cited}

Anderson, W.C. and J.B. Carstens. 1977. Tissue culture propagation of broccoli, Brassica oleracea (italica group), for use in F1 hybrid seed production. J. Amer. Soc. Hort. Sci. 102:6973.

Christey, 1989. Cell and tissue culture studies of Brassica oleracea and B. campestris. PhD Diss., Cornell Univ., Ithaca, N.Y.

Dickson, M.H. 1985. Male sterile persistent white curd cauliflower NY 7642A and-its fertile maintainer NY 7642B. HortScience 20:957.

Dietert M.F., S.A. Barron, and O.C. Yoder. 1982. Effects of genotype on in vitro culture in the genus Brassica. Plant Sci. Lett. 26:233-240.

Dunwell, J.M. 1981. In vitro regeneration from excised leaf discs of three Brassica species. J. Expt. Bot. 32:789-799.

Fly, J., A. Barnason, and R.B. Horsch. 1987. Transformation of Brassica napus with Agrobacterium tumefaciens based vectors. Plant Cell Rpt. 6:321-325.

Jourdan, P.S., E.D. Earle, and M.A. Mutschler. 1989. Atrazine-resistant cauliflower obtained by somatic hybridization between Brassica oleracea and ATR- B. napus. Theor. Appl. Genet. 78:271-279.

Lazzeri, P.A. and J.M. Dunwell. 1986. In vitro regeneration from seedling organs of Brassica oleracea var. italica Plenck cv. Green Comet. I. Effect of plant growth regulators. Ann. Bot. 58:689-697.

Linsmaier, E.M. and F. Skoog. 1965. Organic growth factor requirements of tobacco tissue cultures. Physiol. Plant. 18:100-127.

Narasimhulu, S.B., S. Prakash, and V.L. Chopra. 1988. Comparative shoot regeneration responses of diploid Brassicas and their synthetic amphidiploid products. Plant Cell Rpt. 7:525527.

Robertson, D. and E.D. Earle. 1986. Plant regeneration from leaf protoplasts of Brassica oleracea var. italica cv. Green Comet broccoli. Plant Cell Rpt. 5:61-64.

Stringam, G.R. 1977. Regeneration in stem explants of haploid rapeseed (Brassica napus L.). Plant Sci. Lett. 9:115-119.

Williams, P.H. 1985. Resource book. Crucifer Genetics Coop., Dept. of Plant Pathology, Univ. of Wisconsin, Madison.

Zee, S.Y. and B.B. Johnson. 1984. Cole crops, p. 227-246. In: P.V. Ammirato, D.A. Evans, W.R. Sharp, and Y. Yamada (eds.). Handbook of plant cell culture. vol. 3. Crop species. Macmillan, New York. 